\title{
Introduzione
}

\section{Ilaria Crotti}

Il presente fascicolo di Archivio d'Annunzio, il sesto di una serie che è venuta acquisendo negli anni un profilo di tutto rilievo, nell'alternare a fascicoli dedicati a disamine di tenore comparatistico, storiografico ed estetico altri riservati in misura maggiore a interpretazioni testuali e a verifiche filologiche, interroga una delle forme che contrassegna in misura rilevante la produzione letteraria italiana, e fin dalle sue origini, ossia quella distintasi per brevitas: una scelta che chiama in causa anche sinonimi e antonimi implicati nella forma 'breve' e i significati non univoci che essa veicola.

Ecco che nella sezione Officina dannunziana vari apporti si cimentano proprio in detto ambito, che si rivela estremamente proficuo per sondare non solo il laboratorio ideativo dell'Imaginifico ma anche le relazioni, sia testuali-formali sia biografiche e autobiografiche, poste in essere.

Così, il contributo di Alessandra Trevisan, editando e commentando alcuni pezzi epistolari indirizzati da Matilde Serao a d'Annunzio, ne enuclea i risvolti autobiografici più significativi, atti ad appurare altresì determinate ipotesi interpretative, concernenti le prassi creative saggiate. Cristina Montagnani, da parte sua, conduce una fine lettura filologica intertestuale, non dimentica dei taccuini, del cantiere che il compositore delle Laudi pone in essere, mentre focalizza in particolare Ferrara, Pisa, Ravenna, le future Città del silenzio. Mentre è la prosa del Notturno la tipologia testuale presa in esame, soprattutto in accezione autobiografica, da Federica Maria Giallombardo, sono le Faville del maglio e la linea ermeneutica che le induce a dialogare a distanza ravvicinata con Alcyone il campo d'indagine prescelto da Raffaella Bertazzoli, vagliato con acribia sia filologica che interpretativa.

Grazie alla sezione Civiltà dannunziana il presente fascicolo si impreziosisce di apporti ulteriori. Come quello che si deve a Maria Belponer, la quale nelle sue pagine ha saputo cogliere con sagacia il dialogo incessante che ricorre tra il linguaggio del mito classico ovidiano nel Pascoli dei Poemi conviviali, dei quali Belponer nel 2009 è stata attenta curatrice, per un verso, e nel d'Annunzio dell'Alcyone, per un altro. 
Alfredo Sgroi, da parte sua, esplora con acribia la linea ideologicoestetica che intercorre tra il pescarese e Oswald Spengler, nell'attesa messianica di una figura utopica di uomo d'ordine - un novello Cesare cui spetterebbe il compito di risollevare le sorti della civiltà occidentale, segnate dal drastico declino di ideali intellettuali aristocratici, ritenute pertanto al crepuscolo. Infine, si deve al contributo di Elena Maiolini la formulazione di un bilancio estremamente calibrato degli studi più significativi dedicati alla filologia dannunziana: vera e propria rassegna utile ad arricchire il dibattito più avvertito, pronta com'è a puntualizzarne alcuni snodi metodologici.

A compimento del fascicolo, come di consueto, ecco lo spazio riservato alle recensioni, la prima delle quali destinata da Maiolini al recente volume di Cristina Montagnani e Pierandrea De Lorenzo, Come lavorava d'Annunzio (2018), mentre la seconda da Paolo Parachini a una selezione, attentamente curata da Cecilia Gibellini, delle missive inedite inviate dal consorte a Maria Hardouin, la 'principessa di Montenevoso': Lettere di d'Annunzio alla moglie (2018); in chiusura, il ponderoso ritratto dannunziano ad opera di Maurizio Serra in D'Annunzio le Magnifique, nella recensione di Veronica Tabaglio. 